\title{
Avaliação nutricional, qualidade de vida e risco de sarcopenia em idosos frequentadores de instituições day care de São Paulo e Ribeirão Preto
}

\author{
Nutritional assessment, quality of life and risk of sarcopenia in elderly people attending day \\ care institutions in São Paulo and Ribeirão Preto
}

DOI: $10.37111 /$ braspenj.2020353007

Noeli Aparecida Rosa de Morais'

Renata Furlan Viebig ${ }^{2}$

\begin{abstract}
RESUMO
Objetivo: Investigar associações entre estado nutricional, autopercepção de saúde e risco de sarcopenia em frequentadores de instituições do tipo Day Care, comparando idosos da capital e do interior de São Paulo. Método: Estudo transversal, realizado em duas instituições do tipo Day Care, uma de São Paulo e a outra de Ribeirão Preto, sendo a amostra de conveniência composta por 28 idosos. Foram realizadas entrevistas individuais para obter dados pessoais e a autopercepção de saúde. Para avaliar estado nutricional e risco de sarcopenia foram mensurados dados antropométricos (peso, altura, dobras cutâneas e circunferências corporais) e foi aplicado - questionário SARC-F, traduzido e validado para o português. Para avaliar o risco nutricional dos idosos foi utilizada a Mini-Avaliação Nutricional (MAN) e o apetite dos idosos foi investigado pelo Questionário Nutricional Simplificado de Apetite (QNSA). A análise estatística foi realizada com auxílio do software SPSS v.21, em um nível de significância de $5 \%$. Foi utilizado o teste de qui-quadrado para investigar as associações entre as variáveis obtidas. Resultados: Houve diferença estatisticamente significativa $(p=0,035)$ da autopercepção de saúde entre os idosos das duas cidades, sendo que nenhum idoso da capital considerava sua saúde "excelente". Os idosos do interior apresentaram um índice de massa corporal médio significativamente menor do que o valor encontrado na capital $(p=0,013)$. Diferenças estatisticamente significativas também foram observadas para a circunferência de braço $(p<0,001)$ e dobra cutânea do tríceps $(p<0,001)$. Os resultados da MAN demonstraram risco nutricional para os idosos de ambas as cidades. Por outro lado, as pontuações médias no SARC-F, nas duas cidades, não indicaram risco significativo de sarcopenia. Conclusões: Embora, não tenha sido observado o risco de sarcopenia na maioria da população estudada, os resultados sugeriram que os idosos do interior apresentam melhor percepção de sua saúde, mas com indicadores antropométricos indicativos de déficit nutricional.
\end{abstract}

\section{ABSTRACT}

Objective: To investigate associations among nutritional status, self-perceived health and risk of sarcopenia in people attending Day Care institutions, comparing elderly people in the capital and in the interior of São Paulo. Methods: Cross-sectional study, carried out in two Day Care institutions, one in São Paulo and the other in Ribeirão Preto, with the convenience sample consisting of 28 elderly people. Individual interviews were conducted to obtain personal data and self-perceived health. To assess nutritional status and risk of sarcopenia, anthropometric data (weight, height, skin folds and body circumferences) were measured and the SARC-F questionnaire was applied, translated and validated into Portuguese. To assess the nutritional risk of the elderly, the Mini Nutritional Assessment (MAN) was used and the appetite of the elderly was investigated by the Simplified Nutritional Questionnaire for Appetite (QNSA). The statistical analysis was performed with the aid of the SPSS v.21 software, at a significance level of 5\%. The chi-square test was used to investigate the associations among the variables obtained. Results: There was a statistically significant difference $(p=0.035)$ in self-perceived health among the elderly in both cities, and no elderly person in the capital considered their health "excellent". The elderly in the interior had an average body mass index significantly lower than the value found in the capital $(p=0.013)$. Statistically significant differences were also observed for arm circumference $(p<0.001)$ and skinfold triceps ( $p<0.001)$. MAN's results demonstrated nutritional risk for the elderly in both cities. On the other hand, the average SARC-F scores in both cities did not indicate a significant risk of sarcopenia. Conclusions: Although the risk of sarcopenia was not observed in the majority of the studied population, the results suggested that elderly people in the countryside have a better perception of their health, but with anthropometric indicators indicative of nutritional deficit.

Aceito para publicação

30 de agosto de 2020

1. Graduada em Nutrição, Universidade Presbiteriana Mackenzie, São Paulo, SP, Brasil.

2. Nutricionista, mestre em Saúde Pública (área de concentração -nutrição) pela Faculdade de Saúde Pública da Universidade de São Paulo (USP), doutora em Ciências pelo Departamento de Medicina Preventiva da Faculdade de Medicina da Universidade de São Paulo e Professora adjunta do curso de Nutrição da Universidade Presbiteriana Mackenzie, São Paulo, SP, Brasil. 


\section{INTRODUÇÃO}

O envelhecimento da população é um dos fatos mais importante da sociedade atual, que nos próximos anos trará desafios cada vez maiores. No Brasil, o crescimento da população idosa está ocorrendo de forma acelerada, trazendo, assim, maiores desafios à saúde pública? .

Muitas alterações orgânicas, fisiológicas e metabólicas, relacionadas ao processo normal de envelhecimento, tornam o indivíduo idoso mais vulnerável às alterações da ingestão alimentar, da digestão, absorção e biodisponibilidade de nutrientes e da capacidade funcional, o que pode afetar o estado nutricional do idoso. Dentre as principais consequências do processo de envelhecimento está a perda fisiológica, involuntária e progressiva de massa muscular, conhecida como sarcopenia. A sarcopenia causa redução da força física e da estabilidade do corpo, levando a aumento de fraqueza muscular, limitação de mobilidade e maior risco de quedas, fraturas e lesões².

A qualidade de vida (QV) na velhice tem sido motivo de amplas discussões em todo o mundo, com foco em preservação da saúde e bem-estar global nessa fase da vida. Com base nesta perspectiva, a Organização Mundial da Saúde (OMS) definiu qualidade de vida como a "percepção do indivíduo de sua posição na vida, no contexto da cultura e sistema de valores nos quais ele vive, e em relação aos seus objetivos, expectativas, padrões e preocupações" ${ }^{\prime 3}$.

Existem fortes evidências de que a saúde autopercebida é um excelente preditor da saúde objetiva, ou seja, do número de doenças crônicas, grau de incapacidade funcional e depressão, que resulta em uma conjectura da mortalidade na população idosa ${ }^{4}$. Além disso, aspectos sociodemográficos, como idade, sexo, nível de escolaridade e renda, são alguns dos fatores associados à percepção de saúde encontrados na literatura ${ }^{4}$. Assim, trata-se de um indicador considerado confiável, capaz de expressar aspectos de saúde física, cognitiva e emocional dos indivíduos e vem sendo utilizada em vários estudos.

A percepção da própria saúde é um indicador forte do estado de saúde dos idosos porque prevê de forma consistente a sobrevida dessa população. Como a percepção de saúde se refere a um julgamento subjetivo, ela não pode ser determinada por outra pessoa ${ }^{5}$.

A Política Nacional do Idoso classifica os serviços de atenção à saúde do idoso como serviços de proteção social e serviços de inclusão social. Dentre os serviços de proteção social, inclui-se a modalidade Day Care, que oferece atendimento multiprofissional aos idosos, desenvolvendo promoção e proteção de saúde, bem como incentivando a socialização de seus frequentadores ${ }^{6}$.

Instituições do tipo Day Care para idosos, embora ainda raras, estão se tornando mais comuns nas grandes cidades brasileiras. Nestas instituições, idosos que não necessitam de cuidados especiais de saúde podem conviver, confraternizar, aprender novas atividades, realizar atividades físicas e se alimentar ao longo do dia. Assim, espera-se que impactos positivos da frequência dos idosos aos serviços de Day Care possam se refletir em melhora do estado nutricional, da preservação da capacidade cognitiva, da interação social e da autopercepção de saúde.

Neste panorama, o presente estudo teve como objetivo buscar associações entre o estado nutricional e autopercepção de saúde de idosos, buscando identificar o risco de sarcopenia em frequentadores de instituições particulares do tipo Day Care, da capital e do interior do Estado de São Paulo.

\section{MÉTODO}

Tratou-se de um estudo transversal descritivo, realizado em duas instituições Day Care da capital e do interior de São Paulo, cuja amostra foi composta por conveniência, por frequentadores das instituições referenciadas, homens e mulheres.

Neste estudo, foram considerados elegíveis os idosos com idade igual ou superior a 60 anos. Não foram incluídos no estudo idosos que apresentavam problemas cognitivos e outras condições que os limitassem a participar das etapas do estudo, como surdez, dificuldades na fala ou problemas visuais graves. Como instituições do tipo Day Care para idosos ainda não existem em grande número no país, apenas duas instituições foram convidadas a participar do estudo e nestas, somente 28 frequentadores cumpriram os critérios de seleção e puderam integrar a amostra final da presente pesquisa.

A coleta de dados foi conduzida em dois dias consecutivos em cada uma das instituições, tendo sido realizada entrevista individual com os idosos, com a finalidade de obter informações pessoais, como sexo, estado civil, escolaridade e atividade física.

Quanto ao critério metodológico para a avaliação da autopercepção da saúde, pesquisas têm proposto a categorização da variável adotada no presente estudo de acordo com as seguintes respostas possíveis: ruim, regular, boa e muito boa/excelente ${ }^{7}$.

O estado nutricional dos idosos foi avaliado por meio dos dados antropométricos, como peso, estatura, dobra cutânea tricipital (DCT), circunferência do braço (CB), circunferência da panturrilha $(C P)$ e espessura do músculo adutor do polegar (EMAP).

O peso corporal foi aferido em balança portátil digital da marca Toledo ${ }^{\circledR}$ previamente calibrada, com capacidade de aferição de $130 \mathrm{~kg}$ e a estatura foi obtida com auxílio do estadiômetro da marca Sanny ${ }^{\circledR}$, de acordo com plano de Frankfurt ${ }^{8}$, em triplicata. 
A partir do peso e da estatura obtidos foi estimado o índice de massa corporal (IMC), cujos valores obtidos foram avaliados segundo os seguintes critérios: $<23 \mathrm{~kg} / \mathrm{m}^{2}$, "magreza ou desnutrição", entre 23,1 e $28 \mathrm{~kg} / \mathrm{m}^{2}$, "eutrofia", e acima de $28,1 \mathrm{~kg} / \mathrm{m}^{2}$, "sobrepeso".

As dobras cutâneas foram mensuradas em triplicata, utilizando-se os valores médio entre as medidas. Para a aferição da dobra cutânea tricipital (DCT), foi utilizado o adipômetro da marca Sanny ${ }^{\circledR}$, e foi comparado aos dos percentis propostos no estudo ${ }^{8} \mathrm{~A}$ circunferência do braço (CB) foi mensurada com a fita métrica de aço plano, marca Sanny ${ }^{\circledR}$, sendo os resultados obtidos comparados aos valores de referência eutrofia (> percentil 25) e desnutrição ( $\leq$ percentil 25), de acordo com o estudo 8

Para a aferição da EMAP, que é sugerida como um marcador promissor de massa muscular em condições clínicas ${ }^{9,10}$, foi utilizado o adipômetro marca Sanny ${ }^{\circledR}$. Os indivíduos foram classificados de acordo com os parâmetros propostos por Lameu et al. ${ }^{9}$, que consideram como adequados para homens valores de EMAP $>12,5 \mathrm{~mm}$ e para mulheres, $>10,5 \mathrm{~mm}$.

Para mensurar a circunferência da panturrilha $(C P)$, foi utilizada fita métrica de aço plano, marca Sanny ${ }^{\circledR}$. Resultados inferiores a $31 \mathrm{~cm}$ foram considerados como "depleção de massa muscular", de acordo com o proposto por Vellas et al." .

Para a estimativa da composição corporal dos idosos, foi utilizada a bioimpedância (BIA) perna-a-perna, com auxílio do equipamento do modelo HBF-514C, da marca Omron ${ }^{\circledR}$.

apetite e risco de perda de peso foram avaliados por meio do Questionário Nutricional Simplificado de Apetite (QNSA), o qual se refere à versão curta do Council of Nutricion Appetite Questionnaire $(C N A Q)^{12}$. O escore total do questionário pode variar de 4 a 20 pontos. Quanto menor o escore, maior é o risco de perda de peso. No instrumento original, índices inferiores ou iguais a 14 indicam risco de perda de pelo menos $5 \%$ de peso em seis meses.

risco de desnutrição foi estimado por meio de uma entrevista padronizada, a versão traduzida para o português da Mini-Avaliação Nutricional (MAN), uma ferramenta que pode identificar, em pacientes com idade maior ou igual a 60 anos, presença ou risco de desnutrição ${ }^{13}$. Pontuações finais na MAN de 24 a 30 pontos indicam estado nutricional normal; de 17 a 23,5 pontos apontam que o idoso está sob risco de desnutrição e um escore final menor de 17 pontos indica que o idoso está desnutrido.

Foi aplicado o instrumento SARC-F para triar o risco de sarcopenia, pois este avalia a força muscular, a necessidade da assistência para caminhar, a capacidade de levantar-se de uma cadeira, subir escadas e a frequência de quedas. A pontuação dada a cada item do questionário é de 0 a 2 pontos, podendo chegar à soma de $0 \mathrm{a} 10$ pontos. Pacientes que apresentem um resultado maior ou igual a 4 neste questionário são classificados como risco de sarcopenia ${ }^{14}$.

Para a organização dos dados e a tabulação das informações coletadas foram utilizados o programa Microsoft Excel versão 2013 e, para a análise estatística, foi empregado o pacote SPSS versão 21.0.

Os dados foram avaliados segundo medidas de tendência central (variáveis numéricas) e de acordo com sua distribuição percentual (variáveis categóricas). As associações entre as variáveis obtidas foram realizadas por meio do teste chiquadrado, em um nível de significância de 5\%.

Os procedimentos adotados nesse estudo respeitaram as diretrizes nacionais que regulamenta a ética em pesquisa com seres humanos. Antes da coleta de dados, foi devidamente explicada à instituição e a todos os participantes os objetivos e procedimentos do estudo, participação voluntária e riscos, mesmo que mínimos. Após consentimento do responsável pela instituição e dos sujeitos da pesquisa que consentiram voluntariamente em participar do estudo, o Termo de Consentimento Livre e Esclarecido (TCLE) foi assinado, de acordo com o CAAE: 50839915.9.0000.0084.

\section{RESULTADOS}

A amostra foi constituída de 28 idosos, sendo 14 residentes na cidade de Ribeirão Preto e 14 na cidade de São Paulo. Houve predomínio do gênero feminino, em ambas as cidades, sendo que as idosas representaram $85,7 \%$ da amostra total. A análise descritiva dos dados sociodemográficos dos idosos é apresentada na Tabela 1.

É possível observar que a maioria dos idosos era viúva e todos os participantes, fossem esses do interior ou da capital, relataram praticar algum tipo de atividade física regularmente, com predomínio de 2 vezes na semana (Tabela 1).

Ainda na Tabela 1, observa-se que a maioria dos idosos de São Paulo $(78,6 \%)$ e de Ribeirão Preto $(71,4 \%)$ havia estudado formalmente por até 8 anos (conclusão do ensino fundamental II), sendo que dentre estes, $21,4 \%$ de São Paulo e 28,6\% de Ribeirão Preto tinham mais de 12 anos de estudo (ensino superior).

Foi observada diferença estatisticamente significativa da autopercepção de saúde entre os idosos das duas cidades $(p=0,035)$. Em contrapartida, na cidade de São Paulo, nenhum idoso classificou sua saúde como "excelente", sendo que a maioria dos idosos (93\%) a classificou como "boa" e $7 \%$ como "regular" (Tabela 1). Nenhum idoso classificou sua saúde como sendo "ruim".

Os resultados descritivos das variáveis antropométricas e de composição corporal estão apresentados na Tabela 2. Não foi observada diferença estatisticamente significativa entre a idade média dos idosos das duas cidades. 
Com relação ao estado nutricional, em Ribeirão Preto, pôde-se observar um valor médio de IMC de 22,75 $\pm 2,58$ $\mathrm{kg} / \mathrm{m}^{2}$, indicativo de baixo peso para idosos. Este resultado foi significativamente menor $\left(2,8 \mathrm{~kg} / \mathrm{m}^{2}\right)$ do que o valor encontrado em São Paulo $(p=0,013)$ (Tabela 2).
A classificação dos idosos segundo o IMC revelou que, na cidade de Ribeirão Preto, $57,1 \%(n=8)$ dos idosos tinham baixo peso, $35,7 \%(n=5)$ eram eutróficos e apenas um participante apresentava sobrepeso. Na cidade de São Paulo, apenas $14,3 \%$ dos idosos apresentaram baixo peso, $71,4 \%$

Tabela 1 - Análise descritiva de dados sociodemográficos dos idosos avaliados, nas cidades de São Paulo e Ribeirão Preto, 2017.

\begin{tabular}{|c|c|c|c|c|c|c|c|}
\hline \multirow[b]{2}{*}{ Características } & \multicolumn{2}{|c|}{ São Paulo } & \multicolumn{2}{|c|}{ Ribeirão Preto } & \multicolumn{2}{|c|}{ Total } & \multirow[t]{2}{*}{$p$-valor } \\
\hline & $(n=14)$ & $\%$ & $(n=14)$ & $\%$ & $(n=28)$ & $\%$ & \\
\hline \multicolumn{8}{|l|}{ Gênero } \\
\hline Feminino & 13 & 92,9 & 11 & 78,6 & 24 & 85,7 & \multirow[t]{2}{*}{-} \\
\hline Masculino & 1 & 7,1 & 3 & 21,4 & 4 & 14,3 & \\
\hline \multicolumn{8}{|l|}{ Estado Civil } \\
\hline Solteiro (a) & 2 & 14,3 & 1 & 7,1 & 3 & 10,7 & \multirow{4}{*}{0,233} \\
\hline Casado (a) & - & - & 2 & 14,3 & 2 & 7,1 & \\
\hline Divorciado (a) & 3 & 21,4 & _ & - & 3 & 10,7 & \\
\hline Viúvo (a) & 9 & 64,3 & 11 & 78,6 & 20 & 71,4 & \\
\hline \multicolumn{8}{|l|}{ Escolaridade } \\
\hline Educação infantil & 3 & 21,4 & 1 & 7,1 & 4 & 14,3 & \multirow{4}{*}{0,463} \\
\hline Ensino fundamental & 4 & 28,6 & 2 & 14,3 & 6 & 21,4 & \\
\hline Ensino médio & 4 & 28,6 & 6 & 42,9 & 10 & 35,7 & \\
\hline Ensino superior & 3 & 21,4 & 5 & 35,7 & 8 & 28,6 & \\
\hline \multicolumn{8}{|l|}{ Atividade física } \\
\hline Mais de 2 vezes na semana & 10 & 71,4 & 11 & 78,6 & 21 & 75 & \multirow[t]{2}{*}{ - } \\
\hline Menos de 2 vezes na semana & 4 & 28,6 & 3 & 21,4 & 7 & 25 & \\
\hline \multicolumn{8}{|l|}{ Autopercepção de saúde } \\
\hline Excelente & _ & - & 5 & 35,7 & 5 & 17,9 & \multirow{3}{*}{0,035} \\
\hline Bom & 13 & 92,9 & 9 & 64,3 & 22 & 78,6 & \\
\hline Regular & 1 & 7,1 & _ & $\ldots$ & 1 & 3,5 & \\
\hline
\end{tabular}

Tabela 2 - Análise da relação das variáveis estudadas em idosos frequentadores de um centro de convivência tipo Day Care, nas cidades de São Paulo e Ribeirão Preto, 2017.

\begin{tabular}{|c|c|c|c|c|c|}
\hline \multirow[b]{2}{*}{ Variáveis } & \multicolumn{2}{|c|}{ São Paulo } & \multicolumn{2}{|c|}{ Ribeirão Preto } & \multirow[b]{2}{*}{$p$} \\
\hline & Média & DP & Média & DP & \\
\hline Idade (anos) & 78,143 & 12,0119 & 85,286 & 5,6762 & 0,059 \\
\hline Estatura (m) & 1,566 & 0,0558 & 1,549 & 0,0858 & 0,554 \\
\hline IMC $\left(\mathrm{kg} / \mathrm{m}^{2}\right)$ & 25,534 & 2,9052 & 22,746 & 2,5810 & 0,013 \\
\hline $\mathrm{CB}(\mathrm{cm})$ & 28,821 & 2,6502 & 23,607 & 4,2525 & 0,001 \\
\hline $\mathrm{CP}(\mathrm{cm})$ & 33,571 & 3,2514 & 31,429 & 2,5333 & 0,063 \\
\hline EMAP (mm) & 13,429 & 2,1738 & 13,000 & 2,2871 & 0,616 \\
\hline QNSA (pontos) & 13,8 & 0,9750 & 12,8 & 1,1883 & 0,066 \\
\hline MAN (pontos) & 13,2 & 0,9750 & 11,7 & 1,4899 & 0,005 \\
\hline SARC-F (pontos) & 3,4 & 0,7449 & 3,3 & 1,8576 & 0,895 \\
\hline
\end{tabular}

$\overline{\mathrm{CB}}$ = Circunferência do braço; $\mathrm{CP}$ = Circunferência da panturrilha; DCT = Dobra cutânea tricipital; EMAP = Espessura do musculo adutor do polegar; GORDBIA = Gordura corporal; GORDVISC = Gordura visceral; IMC = Índice de massa corpórea; MAN = Mini Avaliação Nutricional; QNSA = Questionário Nutricional Simplificado de Apetite; SARC-F = Questionário de Sarcopenia. 
$(n=10)$ foram categorizados como eutróficos e $21,4 \%(n=3)$ tinham sobrepeso.

Os idosos de São Paulo apresentaram valores médios de CB e DCT indicativos de normalidade (Tabela 2). Houve diferença estatisticamente significativa entre os valores médios de CB e DCT dos idosos do interior e da capital, sendo que os idosos da cidade de Ribeirão Preto apresentaram, em média, CB reduzida e DCT com risco de depleção.

Os resultados obtidos pela somatória de pontos da MiniAvaliação Nutricional (MAN) demonstraram pontuações indicativas de desnutrição (abaixo de 17 pontos) em ambas as cidades, sendo a pontuação média dos idosos do interior significativamente menor ( $p=0,005)$ (Tabela 2).

Os resultados das pontuações médias obtidas no QNSA, tanto na capital quanto no interior, apresentaram-se abaixo de 14 pontos, especialmente em Ribeirão Preto (12,8 pontos), indicando risco de perda de peso de $5 \%$, em 6 meses.

Com relação à avaliação de sarcopenia, os valores médios de CP e EMAP, tanto na capital quanto no interior, foram considerados adequados, não indicando depleção importante de massa muscular (Tabela 2).

Apenas 9\% dos idosos avaliados, em ambas as instituições, apresentaram pontuação indicativa de sarcopenia no SARC-F. Além disso, as pontuações médias do SARC-F, no interior e na capital, não se mostraram estatisticamente diferentes e não apontaram risco de sarcopenia dentre os idosos, indicando capacidade funcional ainda preservada.

\section{DISCUSSÃO}

No presente estudo, foi possível observar que os idosos do interior paulista apresentaram maior prevalência de baixo peso e risco de desnutrição em comparação aos idosos da capital. Por outro lado, a autopercepção de saúde foi melhor dentre os idosos do interior. Em média, os idosos de ambas as instituições não apresentaram sarcopenia, nem estimada por medidas antropométricas referentes à reserva de massa muscular (CP e EMAP) e nem pela pontuação obtida pelo SARC-F.

Em relação ao gênero, houve prevalência do sexo feminino entre os idosos entrevistados. Resultados semelhantes foram observados em pesquisas realizadas no Brasil, o que se deve, em partes, à maior probabilidade de sobrevivência entre as mulheres no país ${ }^{15}$. Trata-se de um fenômeno denominado "feminização da velhice", associado a fatores que vão do biológico ao cultural.

Uma pesquisa realizada em Passo Fundo, RS, com idosos residentes em uma Instituição de Longa Permanência para Idosos (ILPI), encontrou predomínio de mulheres, assim como no presente estudo, com baixa escolaridade ${ }^{16}$. Os autores também apontaram que a população geriátrica no Brasil apresenta predomínio do sexo feminino e com baixo nível de escolaridade. Da mesma forma, um outro estudo realizado também na cidade de Passo Fundo, RS, demonstrou que houve prevalência do gênero feminino, assim como na presente pesquisa ${ }^{4}$.

A autopercepção do idoso em relação ao seu estado de saúde é um indicador relevante do seu bem-estar, útil para avaliar suas necessidades de saúde e para predizer sua sobrevida ${ }^{3}$. Em estudo feito na cidade de Porto Alegre, RS, a maioria dos idosos relatou estar em boa condição de saúde, mesmo fazendo uso de medicação sistêmica ${ }^{17}$, o que reforça a ideia de que a percepção de ser doente está mais relacionada às incapacidades do que a ser portador de doenças crônicas ${ }^{7}$. Outro estudo gaúcho demonstrou que a autopercepção de saúde pelos idosos foi considerada "boa" por $54 \%$ da população estudada" 18 dados que corroboram os achados do presente estudo, que revelou que, na cidade de São Paulo, 64,3\% dos idosos entrevistados consideram sua saúde "boa". Entretanto, resultados da cidade de Ribeirão Preto demonstraram satisfação ainda maior dos idosos com sua saúde, pois a grande maioria dos entrevistados $(92,9 \%)$ considera-se com "boa" saúde.

Houve diferença importante entre os valores de IMC encontrados nos idosos da capital e no interior, sendo que os de Ribeirão Preto apresentavam uma prevalência de baixo peso 42,8\% maior que na capital. A prevalência de baixo peso apontada pelo IMC, na cidade do interior $(57,1 \%)$, foi semelhante à encontrada em um estudo realizado com idosos institucionalizados do Estado de São Paulo, no qual foi observado que pouco mais da metade da população avaliada (55\%) apresentou-se na faixa de eutrofia, $27 \%$ com excesso de peso e $18 \%$ apresentaram-se no estado de magreza ${ }^{19}$.

Não houve diferença estatisticamente significativa entre as medidas de CP dos idosos da capital e do interior, sendo que, em ambos os locais, os idosos apresentaram, em média, medidas consideradas normais ( $\geq 31 \mathrm{~cm})$, ou seja, sem perda de massa muscular. A CP representa a medida mais sensível do componente corporal de massa muscular em idosos. Essa medida indica alterações na massa magra que ocorrem com o decréscimo da idade e atividade física ${ }^{20}$.

No presente estudo, idosos da capital apresentaram valores de DCT e CB indicativos de normalidade. Em estudo realizado para avaliação de $\mathrm{DCT}$, em Florianópolis-SC21, com 167 idosos institucionalizados, encontraram-se valores médios de 25,1 mm, o que se assemelha aos resultados encontrados nos idosos da capital do presente estudo (24 $\mathrm{mm}$ ). Por outro lado, os valores médios dos idosos de Ribeirão Preto foram inferiores aos de São Paulo e aos estudos de Florianópolis. 
Os valores médios de EMAP obtidos na presente pesquisa foram indicativos de adequação da massa muscular. Resultados de medidas da EMAP superiores aos do presente estudo foram obtidos em trabalho realizado no Rio Grande do Sul22 com 48 idosos, cujo valor médio foi de 18,59 $\pm 3,95 \mathrm{~mm}$. A EMAP é um parâmetro ainda pouco aplicado e testado na prática de estudos populacionais e, principalmente, com idosos praticantes de atividade física, sugere-se cautela no uso e na interpretação dessa medida, recomendando-se sempre aliá-la a outros parâmetros antropométricos.

Os resultados obtidos pela somatória de pontos da MiniAvaliação Nutricional (MAN) demonstraram pontuações indicativas de risco significativo de perda de peso em ambas as cidades, sendo a pontuação média dos idosos do interior significativamente menor ( $p=0,005)$.

Em um estudo que utilizou a MAN ${ }^{23}$, com idosos vivendo em comunidade portuguesa, foi identificado que apenas $10,5 \%$ de idosos estavam desnutridos, demonstrando um percentual menor de desnutrição do que estudos realizados com indivíduos hospitalizados e institucionalizados de Portugal. Os autores apontaram que a MAN representa um instrumento que revela um resultado mais complexo da avaliação nutricional do idoso em instituições tipo Day Care, pois estas instituições se fazem cada vez mais presente na comunidade. Além disso, a utilização da MAN em espaços como esses é capaz de contribuir, indicando a necessidade de intervenção precoce, e pode ser entendida como parte do processo de promoção da saúde desses indivíduos que frequentam instituições tipo Day Care.

A prevalência de sarcopenia dentre os participantes do presente estudo (9\%) foi bastante inferior em relação a percentuais encontrados por outros autores em estudo na cidade do México (entre 13\% e 24\%) ${ }^{24}$. Em uma outra pesquisa feita com idosas institucionalizadas, residentes de uma ILPI do sul do Brasil, a prevalência de sarcopenia foi de $51 \%{ }^{21}$.

As pontuações médias obtidas pelo questionário SARC-F não indicaram risco para sarcopenia em idoso de nenhuma das cidades. Esta menor prevalência de sarcopenia encontrada no presente estudo pode estar relacionada à prática de atividade física regular dos participantes da presente pesquisa e ao fato de que não estão institucionalizados, mas frequentavam o Day Care e participavam ativamente das atividades físicas, artísticas e educativas propostas.

No presente estudo, as pontuações médias do Questionário Simplificado de Apetite (QNSA), para ambas as cidades, indicaram risco de perda de peso maior que $5 \%$ em 6 meses. Embora pouco empregado no Brasil, o questionário QNSA é importante como avaliação inicial de rotina e facilita uma eventual intervenção nutricional ${ }^{25}$. Ademais, é relevante para o monitoramento dos indivíduos em instituições, e hospitais ou durante intervenção de saúde em programas na comunidade. Assim, a utilização desta ferramenta é importante, visto a perda de apetite e, consequentemente, perda de peso serem condições frequentes e eventos importantes em indivíduos adultos e idosos ${ }^{11}$

Embora os valores médios de IMC dos idosos de São Paulo tenham sido superiores aos observados em Ribeirão Preto, não foram observadas diferenças significativas entre os valores médios de percentual de gordura corporal, mensurado por bioimpedância (BIA). Entretanto, em média, idosos de São Paulo e de Ribeirão Preto apresentaram percentuais de gordura maiores do que 30\% da massa corporal total. É importante considerar que há limitações em se utilizar apenas os valores de bioimpedância do hemicorpo inferior para avaliar a composição corporal e que níveis de hidratação menores, comuns entre idosos, podem influenciar os resultados de percentual de gordura corporal nesta população.

Não foram encontradas, na literatura, pesquisas que tivessem realizado a comparação entre os três métodos subjetivos utilizados no presente estudo para avaliar o estado nutricional de idosos (MAN, QSNA e SARC-F). Resultados das pontuações médias da MAN e do QNSA demonstraram-se mais sensíveis no diagnóstico de risco de desnutrição/perda de peso corporal, evidenciando que, em média, tanto os indivíduos do interior quanto os da capital apresentaram risco para perda de peso. Por outro lado, resultados do SARC-F e das medidas de EMAP e CP, os quais visam encontrar depleção de massa magra/muscular, não demonstraram, em média, o risco de sarcopenia em participantes de ambas as cidades.

\section{CONCLUSÃO}

No presente estudo, não foi observado o risco de sarcopenia para a maioria da população estudada, sejam estes os idosos da capital ou do interior de São Paulo. Entretanto, os resultados obtidos na avaliação antropométrica, pela aplicação da MAN e do QNSA, sugeriram risco de perda de peso dentre os idosos, tanto nos indivíduos da instituição Day Care da cidade de São Paulo como na da cidade de Ribeirão Preto, com resultados da avaliação do estado nutricional já apontando que metade dos idosos do interior estava abaixo do peso.

Por outro lado, os idosos de Ribeirão Preto demonstraramse mais satisfeitos com sua saúde e qualidade de vida do que os da cidade de São Paulo, mesmo que apresentassem maior prevalência de baixo peso e maior risco de desnutrição.

Ressalta-se, de tal forma, que com o avanço do envelhecimento populacional e o aumento do número de Instituições do tipo Day Care, atrelados à possibilidade aumentada de problemas nutricionais neste grupo, torna-se essencial para a saúde do idoso o reconhecimento e a implantação de intervenções precoces nas irregularidades do estado nutricional 
no idoso, para propiciar, além de menores taxas de morbimortalidade, maior grau de satisfação com a própria saúde e melhora da percepção de qualidade de vida.

\section{REFERÊNCIAS}

1. Dantas EL, Brito GEG, Lobato IAF. Prevalência de quedas em idosos adscritos à estratégia de saúde da família do município de João Pessoa, Paraíba. Rev APS. 2012;15(1):67-75.

2. Deutz NE, Bauer JM, Barazzoni R, Biolo G, Boirie Y, BosyWestphal A, et al. Protein intake and exercise for optimal muscle function with aging: recommendations from the ESPEN Expert Group. Clin Nutr. 2014;33(6):929-36.

3. Khoury HTT, Sá-Neves AC. Percepção de controle e qualidade de vida: comparação entre idosos institucionalizados e não institucionalizados. Rev Bras Geriatr Gerontol. 2014;17(3):553-65.

4. Jerez-Roig J, Souza DLB, Andrade FLJP, Lima Filho BF, Medeiros RJ, Oliveira NPD, et al. Autopercepção da saúde em idosos institucionalizados. Ciência \& Saúde Coletiva. 2016;21(11):3367-75.

5. Busato MA, Gallina LS, Teo CRPA, Ferreti F, Pozzagnol M.Autopercepção de saúde e vulnerabilidade em idosos. Rev Baiana de Saúde Pública. 2014;38(3):625-35.

6. Franciulli SE, Ricci NA, Lemos ND, Cordeiro RC, Gazolla JM. The Geriatric Day-Care assistance mode: functional effects in a six month multidisciplinary health care program. Cienc Saude Colet. 2007;12(2):373-80.

7. Borges AM, Santos G, Kummer JA, Fior L, Molin V, Wibelinger LM. Autopercepção de saúde em idosos residentes em um município do interior do Rio Grande do Sul. Rev Bras Geriatr Gerontol. 2014;17(1):79-86.

8. Lebrão ML. O projeto SABE em São Paulo: uma visão panorâmica. In: Lebrão ML, Duarte YAO, eds. SABE: saúde, bem-estar e envelhecimento. O Projeto SABE no Município de São Paulo: uma abordagem inicial. Brasília: Organização Pan-Americana de Saúde; 2003. p.33-43.

9. Lameu ED, Gerude MF, Corrêa RC, Lima KA. Adductor pollicis muscle: a new anthropometric parameter. Rev Hosp Clin Fac Med Sao Paulo. 2004;59(2):57-62.

10. Gonzalez MC, Duarte RR, Budziareck MB. Adductor pollicis muscle: reference values of its thickness in a healthy population. Clin Nutr. 2010;29(2):268-71.

11. Vellas B, Garry PJ, Guigoz Y. The Mini Nutritional Assessment (MNA): research and practice in the elderly. Switzerland: Karger; 1999.
12. Wilson MM, Thomas DR, Rubenstein LZ, Chibnall JT, Anderson S, Baxi A, et al. Appetite assessment: simple appetite questionnaire predicts weight loss in community-dwelling adults and nursing home residents. Am J Clin Nutr. 2005;82(5):1074-81.

13. Hengstermann S, Nieczaj R, Steinhagen-Thiessem E, Schulz RJ. Which are the most efficient items of mini nutritional assessment in multimorbid patients? J Nutr Health Aging. 2008;12(2):117-22.

14. Parra BFCS, Matos LBN, Ferrer R, Toledo DO. SARCPRO: proposta de protocolo para sarcopenia em pacientes internados. BRASPEN J. 2019;34(1):58-63.

15. Almeida AV, Mafra SCT, Silva EP, Kanso S. A feminização da velhice: em foco as características socioeconômicas, pessoais e familiares das idosas e o risco social. Textos \& Contextos. 2015;14(1):115-31

16. Zanin C, Candido JB, Jorge MSG, Wibelinger LM, Doring M, Portella MR. Sarcopenia e dor crônica em idosas institucionalizadas. Br J Pain. 2018;1(4):288-92.

17. Paskulin LMG, Vianna LAC. Perfil sociodemográfico e condições de saúde auto-referidas de idosos de Porto Alegre. Rev Saúde Pública. 2007;41(5):757-68.

18. Pilger C, Menon MH, Mathias TAF. Características sociodemográficas e de saúde de Idosos: contribuições para os serviços de saúde. Rev Latino-Am Enferm. 2011;19(5):1230-8.

19. Galesi LF, Lorenzetti C, Oliveira MRM, Fogaça KCP, Merhi VL. Perfil alimentar e nutricional de idosos residentes em moradias individuais numa instituição de longa permanência no leste do estado de São Paulo. Alim Nutr. 2008;19(3):283-90.

20. Frank AA, Soares EA. Nutrição no envelhecer. São Paulo: Atheneu; 2004.

21. Rauen MS, Moreira EAM, Calvo MCM, Lobo AS. Avaliação do estado nutricional de idosos institucionalizados. Rev Nutr. 2008;21(3):303-10.

22. Cabrera TP, Marcuzzo ML, Kirsten VR. Perfil nutricional de idosos de uma instituição geriátrica de Santa Maria - RS. Disc Scientia: Ciênc Saúde. 2008;9(1):69-76.

23. Santos ALM, Amaral TMSPF, Borges NPGFB. Subnutrição e fatores associados em uma comunidade de idosos Portugueses. Rev Nutr. 2015;28(3):231-40.

24. Baumgartner RN, Koehler KM, Gallagher D, Romero L, Heymsfield SB, Ross RR, et al. Epidemiology of sarcopenia among the elderly in New Mexico. Am J Epidemiol. 1998;147(8):755-63.

25. Rolland Y, Perrin A, Gardette V, Filhol N, Vellas B. Screening older people at risk of malnutrition or malnourished using the Simplified Nutritional Appetite Questionnaire (SNAQ): a comparison with the Mini-Nutritional Assessment (MNA) tool. J Am Med Dir Assoc. 2012;13(1):31-4.

Local de realização do estudo: Universidade Presbiteriana Mackenzie, São Paulo, SP, Brasil.

Conflito de interesse: Os autores declaram não haver. 